\title{
An Agent System for Intelligent Situation Assessment
}

\author{
Qiang Yang \\ School of Computing Science \\ Simon Fraser University \\ Burnaby, BC Canada V5A 1S6 \\ email: qyang Ges.sfu.ca \\ Irène Abi-Zeid and Luc Lamontagne \\ Defence Research Establishment Valcartier \\ Decision Support Technology \\ 2459, boul. Pie XI, nord \\ Val Belair, Quebec, Canada, G3J IXS \\ email: irene.abi-zeid@drev.dnd.ca \\ luc. lamontagne $@$ drev.dnd.ca
}

\begin{abstract}
Coordinating Search and Rescue (SAR) operations is a knowledge and information intensive task. Upon receiving an initial indication about a possible aircraft related problem, a Rescue Coordination Center (RCC) Controller sets out to find out more about the nature of the problem. This situation assessment phase is highly complex duc to the diverse and sophisticated nature of the many information sources. In this paper, we present an intelligent agent architecture incorporating multiple, continual information planning agents for assisting the RCC controller in performing critical situation assessment tasks. The agents monitor the actions of the human controller and make their decisions on when and how to acquire more information to help the human controller, and to remind him of important steps that may have been overlooked. The system is designed based on several technologies, including hierarchical task networks, case based retrieval and intelligent agent systems.
\end{abstract}

\section{Introduction}

Coordinating Search and Rescue (SAR) operations is knowledge intensive. Upon receiving information about a possible air incident (for example, a plane crash or an overdue airplane), a Rescue Coordination Center (RCC) Controller sets out doing detective work, to determine the cause and context of the incident. This work often involves checking on different information sources such as air traffic controllers, salellite data and emergency locator transmitter (ELT) signals, as well as the relatives of the pilot involved. To make the process even more complex, a controller often deals with more than one case at a time, requiring constant re-prioritization of tasks and resources. In the course of all these activities, it is likely that an important task will be overlooked by even an experienced SAR controller.

In this paper, we focus on a portion of the RCC controller's tasks, mainly the situation assessment (SA) aspects for air-related incidents (Air SAR). Related research on knowledge acquisition aspects for the search and rescue operations has been conducted in [Cottom et al] and [Cottom and Shadbolt]. Furthermore, [Lamontagne $e t$ al. 1995] have proposed models for coordinating the activities of multiple RCC controllers using Distributed AI techniques. Situation assessment, a process which is similar to diagnosing a patient by a doctor in the medical diagnosis domain, refers to the tasks of finding out the true nature of the incident and the context information such as the seriousness and the location of the incident. In this paper, we describe our work in sludying the domain and in designing a problem solving model for building an intelligent multi-agent system to assist the air SAR controller in situation assessment. Our conclusion is that the SAR domain is best served with an agent model whereby the agents continuously monitor the coordinator and the information sources as a case unfolds, and provide user-friendly messages to the human conlroller when he/she misses some critical actions in solving a case. Our agent model is based on a combination of analogical reasoning based on similarities [Leake 1996] and Hicrarchical Task Network (HTN) planning techniques [Wilkins 1988], [Tate 1977] and [Yang 1997]. Analogical rcasoning is advantageous in approximate reasoning from the current situations in real time, and is good in dcaling with loosely structured descriptions of domain knowledge. Hierarchical task network based planning, on the other hand, provides a formalism for organizing tasks and their relations in a hierarchical and temporal framework. The combination of these two lechniques provides an underlying model for solving our situation assessment problem. In this system, we use cases to describe hypotheses and to encapsulate information-gathering queries for 
identifying the correct hypothesis. The information gathering task is known as "Communications Search" (COMSEARCH) in the SAR domain. Once a hypothesis is identified as a candidate, a task network model for the information gathering queries encapsulated within the case is retrieved. This model is used to monilor the actions of the human operator, and critical steps are presented to the user at the right time and in a non-intrusive manner. These messages allow the controller to gain more confidence about the case or to overrule the particular hypothesis as unlikely. The process continues in the background until a final conclusion about the nature of the incident is reached by the controller. In this manner, the agent is goal-directed, autonomous and capable of learning new knowledge to adapt itsclf.

In the next section, we describe the Air SAR domain and motivate our work. Subsequently, we describe the clements of the agent model and present examples to highlight important aspects of the model.

\section{Situation Assessment in Search and Rescue Operations}

The rescue coordination center (the $\mathrm{RCC}$ ) is typically manned by two air controllers and two marine controllers. Their roles are to coordinate, control and conduct search and rescue operations in Canada's area of responsibility [NSM95]. In general, an Air SAR operation can be broken down into three phases, where each phase can somewhat overlap the adjacent ones. First, when a call is received (first notice), the operation enters an uncertainty phase. In this phase the air SAR controller will collect the information about the details of the flight plan and people involved. A request may be sent out to different agencies, airports and vessels in response to an ELT signal for more information to verify the exact location and cause of the signal. In this phase the air SAR controller conducts what is known as the communication search using telecommunication channels such as the telephone or satellite information to obtain more detail. If not enough information has been obtained, the operation will progress into the alert phase whereby the operators will expand the search area and means and alert various search and rescue agencies.

If a certain amount of time has passed after the uncertainty phase without obtaining more information about the plane, the operation enters the distress phase. In this phase the air SAR controller should develop a search plan and task any additional resources to assist in the plan. Finally, when the details of the incident are known, a rescue plan must be developed and monitored by the air SAR controller. A case report will be filed in the end, and news dispatches will be sent out to various media agencies before the case is closed.

A first indication of trouble can be signaled by a phone call reporting on an overdue personnel, an ELT (emergency locator transmitter) report received by a high-flying aircraft, a satellite or an airport tower operator. In addition, a search and rescue satellite may report the reception of an ELT signal when it passes over the area in question.

The situation assessment aspect (corresponding to the uncertainty and alert phases) of the controller's tasks is much like detective work. Over $90 \%$ of the cases are false alarms, caused by faulty equipment, power lines or even ELT's that the pilots forgot to turn off. Furthermore, the recejpt of reports on an overdue aircraft, does not necessarily imply that the aircraft itself has crashed somewhere. Rather, there are many possibilities why the craft has gone missing, ranging from the fact that the pilot may have landed halfway to refuel, to the possibility that the pilot never took off in the first place. If the controller becomes convinced that the missing plane is in distress, then he may initiate the tasks associated with the distress phase which consists of planning, coordinating, and monitoring search missions. These tasks are not described in this paper.

Once the cause of the incident and the location of the aircraft are determined, an air SAR controller may be required to mobilize and monitor the rescue process. Tasks in this phase involves notifying various agencies involved, including hospitals and police, notifying the relatives and dispatching planes or helicopters to the crash site.

An Air SAR case often requires extensive investigation and complex hypothesis generation. Here is an example.

An Air SAR controller receives a call from an airport radar operator, reporting on a plane making erratic moves. The operator said that the pilot phoned the control tower to report the wind shield being hit by an object.

The controller wants to get first hand information, so he phones the control tower directly. The tower repeats the information given by the radar operator, and adds that the pilot didn't sound excited, but rather sleepy. This indicates to the controller that it is likely that the pilot had a stroke.

The controller then immediately issues a broadcast, calling for information on missing aircraft to all airports in the area.

(Some details omitted.)

The controller then decides to dispatch a search aircraft and six vessels because the suspected incident area involves a lake. The controller keeps constant contact with the search crew.

(more details omitted.)

This case is more complicated because the information requested by the controller went out on several channels. Additional information from the air traffic control tower indicated the possibility of a stroke, which prompted for further actions by the controller. In this example, the situation assessment aspects are the hypotheses formation 
and elimination for the cause of the incident (stroke), and the communications search plan developed for the retained hypothesis (crash due to stroke)..

We propose to design an intelligent agent system to monitor and assist the air SAR controllers in their situation assessment work. We have gone through interviews and domain model studies to clearly understand the domain. Our understanding has led us to the belief that the resulting system will be an autonomous agent running in the background performing tasks such as monitoring the COMSEARCH activities of the controller and various information sources, providing assistance in the form of reminders and checklists for critical information gathering tasks that might have been overlooked. If necessary, the agent will make the current candidate hypothesis list explicit for the controller who is often overloaded with a vast amount of information, including satellite data, phone calls, data base checks, etc.

Our agent-based system will benefit the SAR controller in several ways. First, because the agent is constantly monitoring a variety of information sources, it can help filter out a large quantity of irrelevant information, and help the air SAR controller concentrate on the critical information only. Second, given the overwhelming workload during the high seasons for air incidents, the agent system can help improve the accuracy and shorten the time required for assessing a case. This effect translates directly into one of saving more lives. Third, for junior air SAR controllers, the agent based system can become a handy decision support system and a tutoring system. We expect that the learning speed of the new controllers will be improved with the help of our agent system.

\section{Agent Model}

\subsection{A Process Model}

The primary aim of our agent system is to plan the information queries. In this regard its goal is similar to the multi-agent system by [Knoblock and Ambite,1996]. The action and query model in our proposal bears similarity with the CHARADE system designed for fighting forest fires [Perini and Ricci, 1996], with a major difference being that our system is mainly aimed at situation assessment as opposed to resource and strategic planning. The manner in which we envision our agent system to function is as follows: First, upon receiving an initial indication of a problem, the available relevant information is input into the system. The system retrieves a collection of similar hypothesis objects constructed from previous situations having similar initial information. These hypothesis objects correspond to different scenarios describing the situation. These consist of the hypotheses $\mathrm{H}_{1}$, $\mathrm{H}_{2}, \ldots \mathrm{H}_{n}$ which can be used to characterize the current situation, where each of the $\mathrm{H}_{1}$ 's provides a plausible cause for the current case such as "plane crashed". Subsequently, the system enters a cycle in which it identifies (from the case base) the communications search tasks described by the task network associated with each hypothesis object, performs these tasks (or recommends to the user the execution of some of these tasks), and then uses the results to rank and retrieve the current and new hypotheses. The overall workflow for this iterative process is depicted below.

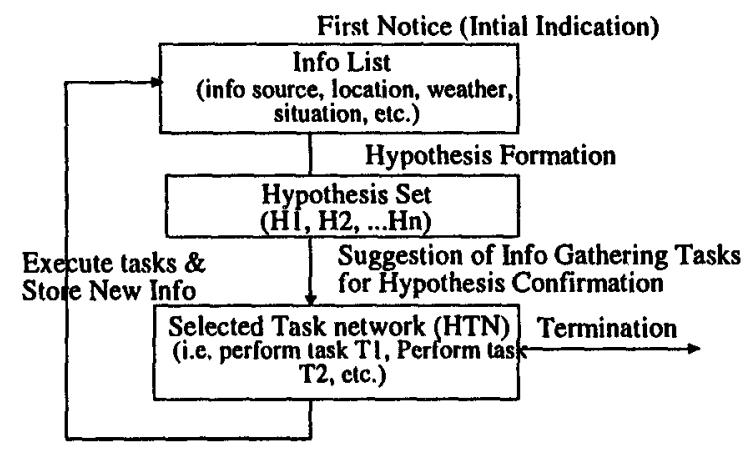

Figure 1: Information flow

In our model, each possible hypothesis is described as an object. The overall system operation is centered around the evaluation and exploration of a hypothesis. Here a hypothesis object is similar to an object in object-oriented technology. It consists of both the problem attributes used to describe the context of a situation and the associated hypothesis-evaluation and task-suggestion methods to operate on them.

In particular, a hypothesis object consists of the following elements: 
- a hypothesis for the cause of the incident

- a hierarchical task network (HTN) for the communications search used to confirm the hypothesis and assess the situation

- a record of executed communications search steps for the current hypothesis object, and the communications search steps yet to be executed for further confirming the hypothesis

- an evaluation function of priorities for not-yet execuled communications search tasks

- indices with weights attached to the expected values of the answers to the queries that can be used to provide a similarity based ranking for the hypothesis object.

Below, we explain some of the key components of a hypothesis object.

\subsection{Hypotheses}

A hypothesis is a plausible cause and outcome for the case. The space of hypotheses naturally forms a hierarchy. In the Air SAR domain, some of the hypotheses are listed below:

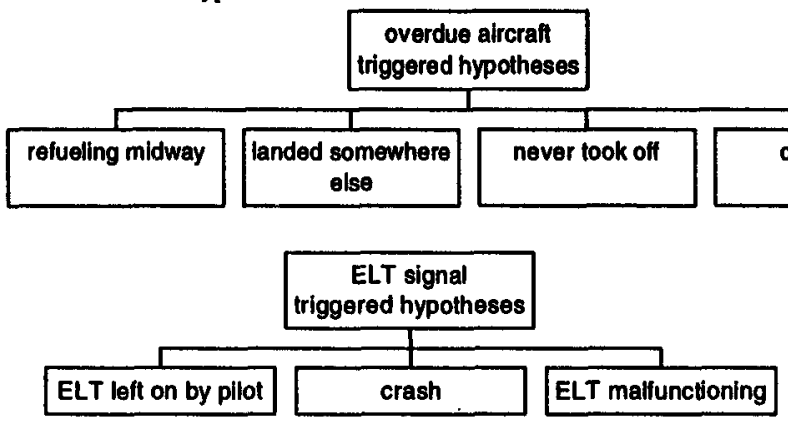

Figure 2: Hypotheses hierarchies

The situation assessment phase of the SAR operation involves finding out which of the hypotheses holds for the current situation, as well as the context, such as the number of people on board and the seriousness of the problem, in which such a hypothesis holds. Initially none of the hypotheses relevant to the current case can be ruled out. More information will be requested by the RCC controller in order to narrow down the hypotheses space. For example, an air SAR controller may check the weather condition to see whether the likelihood of a crash is high given an overdue report. At the same time, a request will be sent out to get the flight plan of the pilot in order to find all airports where the pilot might have landed, and so on.

\subsection{Hierarchical Task Networks}

The basic structure of the situation assessment part of the RCC controller's tasks can be broken down into a hicrarchically structured set of tasks. At the very high level, the tasks correspond directly to the steps defined in the standard operation procedures such as the National SAR Manual [SAR 95]. At the next level down, the tasks are broken into several detailed sub-steps. An example of the top two levels of the hierarchy associated with situation assessment is presented below.

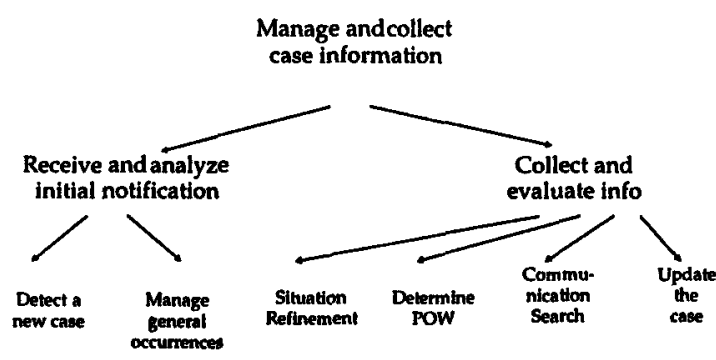

Figure 3: Hierarchical task networks for situation assessment 
Although situation assessment for all cases follow the same pattern of tasks as presented above, the lower levels of the task networks associated with the communications search efforts differ depending on the hypothesis of the incident involved. For example, the communications search task hierarchy triggered by an overdue aircraft report has a different, although overlapping, set of tasks from those involved with an ELT signal case.

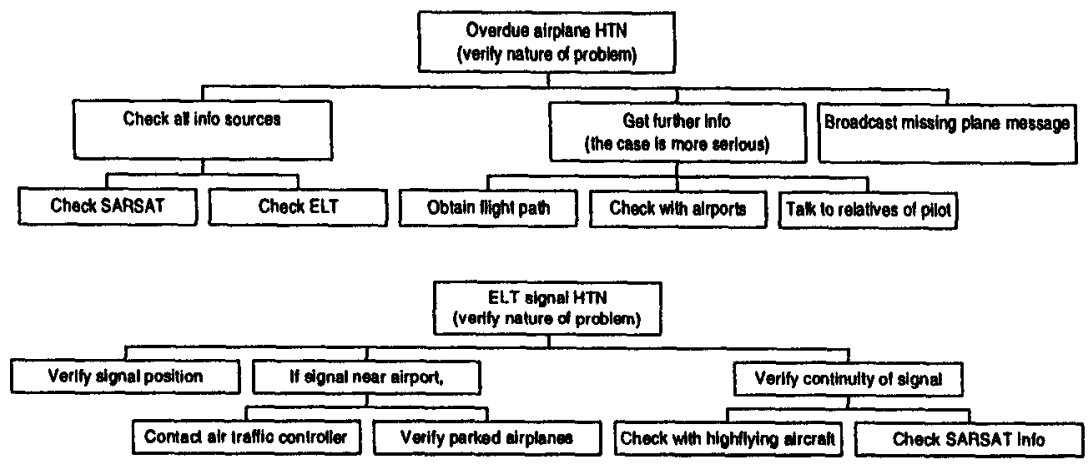

Figure 4: Two different HTN's for different triggers

These two task networks are separately associated with different hypothesis objects. For example, the ELT task network is associated with an ELT related hypothesis such as ELT-malfunction and crashing-aircraft. Similarly, the task network for overdue air crafts is associated with Overdue hypotheses, including overdue-due-to-crash and overdue-landing-elsewhere hypotheses.

Often a task in a task network has more than one way of being accomplished. Take for example the task "verify parked airplanes", a task highlighted in a shaded box in the ELT hierarchy. This task can be accomplished in a number of ways, including either checking with the airport security authority or checking directly with the air traffic control tower. Which particular method to use for accomplishing a particular task depends on the cost of using the method, the reliability of the information source, and potential delays and the availability of the resources. Therefore, a particular function of the agent is to plan the method in which to expand a task in order to maximize the information gain in a minimal time frame under the circumstances. The space in which the agent functions has an AND/OR structure, and furthermore, the agent should be ready to re-plan dynamically should a particular information source become unavailable.

\subsection{Indexes and Their Weighted Connections}

For the RCC controller to assess the current situation, rank the remaining hypotheses, and weigh the next steps, a channel must exist between the known facts and knowledge, and the system stored HTN's and hypotheses. This channel is provided by a layer of indexes. The index structure is very similar to that of a case based reasoning system; its main purpose is to enable a system to perform approximate and similarity based reasoning, a key part of CBR. A key issue here is real-time performance.

In a hypothesis retrieval and ranking cycle, the indexes located on the first level have connection weights that can directly affect the result of matching and ranking the hypotheses on the second level. The process of retrieval is very similar to that in a case based reasoning process, in which a CBR retrieval process scans a case base for the most similar cases to a new problem, ranks the high ranking hypotheses in response to the input index values. For cach candidale hypothesis object, shown in the figure 5 as a box, an evaluation of its similarity with the current situation will be conducted. This evaluation will take into account the weights of the matching indexes. The highest ranked cases will then be presented to a task manager for selecting the next tasks to perform. In the SAR domain, the indexes for a similarity based evaluation can be performed based on the past cases and the current problem instance which is a SAR case with all its known facts and unknown facts. We consider all the tasks in a task network to be potential indexes to any hypothesis object in the HTN library. An information gathering task can be seen as having the following structure: "Get info on TOPIC from SOURCE". In this regard, a task to be performed (combination of TOPIC and SOURCE) and an index are inter-changeable. The outcome of a task corresponds to the different values of a index (answer to the TOPIC from the SOURCE). Each time a the execution of task (query) obtains a value (answer), the score for the related hypothesis is correspondingly reevaluated. 


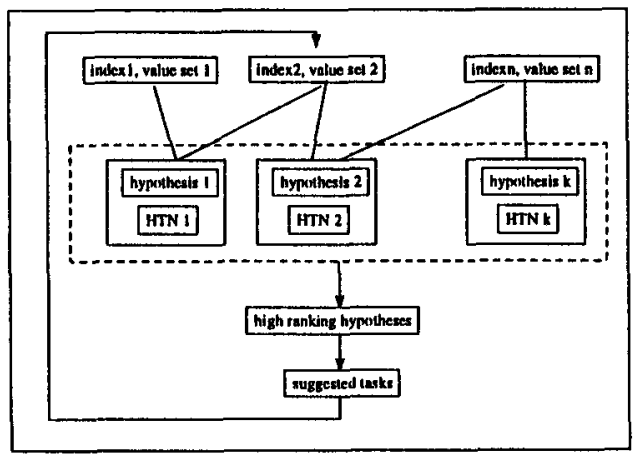

Figure 5: hypotheses ranking and indexing

As an example, consider the task of checking SARSAT for an overdue aircraft information. The task "check SARSAT for overdue aircraft" is an index to the case that can take on the following values: received signal once; continuous signal; no signal; no continuous signal. Each value will provide a different weight for the case. For example, if the value "received continuous signal" is obtained for the index, then the index should contribute to a "plane crashed" hypothesis with a weight higher than that for "received signal once", and so on. In the next section we will discuss how these indexes and their weights are integrated to compute an overall ranking for the case.

\section{Agent Design and Information Flow}

\subsection{Overall Architecture}

The overall objective of our agent system is to assist the controllers at the RCC in dealing with complex tasks. As an assistance system, the goal is the same as that for the overall intelligent agents research--- to design assistance systems that act autonomously, to be goal oriented, and to have the ability to learn from its own experiences and from the interaction with the user.

Our design goal is for the system to be incrementally more competent by integrating a variety of intelligent techniques including case based reasoning, rule based reasoning, HTN planning, process monitoring, active databases and machine learning. Each agent will have its own set of goals, implemented based on active dalabase technology [Widom and Ceri, 1996]. A schematic diagram is shown in the following figure.

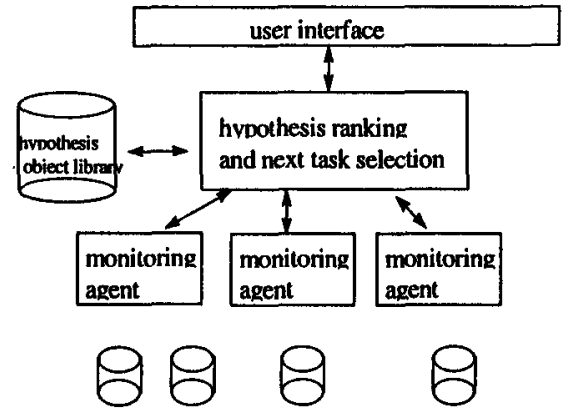

Information sources: databases, SARSAT, ELT, ctc.

Figure 6: agent system components 


\subsection{Task Monitoring}

Having discussed the indexes and their weights, we now explore how to obtain the information about the index values themselves. What we envision is a monitoring agent for each source of information. For example, we can attach one agent for SARSAT dalabase to provide a layer of translation between the SARSAT signal and the task network based indexes. Similarly, another agent is designed to be in charge of monitoring the telephone information source, keeping a tap on calls made to different agencies and resources, and calls received from other telephones. At the Canadian Rescue Coordination Centers, a case tracking system known as the CaseMaster has already been developed. The aim of this system is to replace the hand-written logs that the controllers use for recording all the major steps and tasks they perform. The CaseMaster thus provides an jdeal channel for interaction with our agent system.

Moreover, certain information can be obtained directly from the databases using the active database technology, by assigning triggers to various data sources which can provide information based on an event-based system. Where automatic means are not available for obtaining the current value of a particular index, we can prompt the users through the CaseMaster system for obtaining the answers. This data is then useful for both statistical case tracking and for real time evaluation of the case score.

\subsection{Similarity Based Retrieval of Hypotheses Objects}

When a first evidence of an incident (first notice) is received, a process of hypotheses evaluation would begin. The initial evidence, or the evidence set would be translated to a set of index values for various tasks and subtasks in the task networks associated with different cases in a case base. Using these networks, a ranking process would determine the most promising cases, in terms of similarity to the current situation, given the current problem solving context, such as weather conditions and time of day. The ranking would continue, making some cases more likely than others at any given time, until a final hypothesis or collection of hypotheses is confirmed. A number of strategies have been proposed in the CBR literature for case-retrieval algorithms. Some of them are serial, while others are parallel; Some use flat-indexed structures, but others use hierarchical-indexed structures. Some use indices to construcl structures that distinguish cases from each other at a very small granularity while others discriminate cases at a more coarse level. Each method has its advantages and disadvantages. For example, for a flat case base the best case will be retrieved, but if the case base is very large, the search would be very timeconsuming. On the other hand, if a case base is organized into a tree structure, the search cost is relatively small, hut there is no guarantec that the most similar cases will be retricved. There is always a trade-off between these two extremes.

One of the most popular methods is the Nearest Neighbor Algorithm. It assumes that each case in a case base is defined or indexed by a set of $n$ (numeric or symbolic) features. Given an input query $Q, k-N N$ retrieves a set of $k$ cases most similar to $Q$ from the case base. These $k$ cases have the least distance from $Q$. For a case $C$ and a query $Q$. The following formula is used to compute a similarity measure between two a case $C$ and input query $Q$, in terms of the similarity between two indexes or features:

$\operatorname{sim}(C, Q)=\operatorname{norm}^{*}\left(\sum\left(W_{i}^{*} \operatorname{sim}\left(f_{i}^{c}, f_{i}^{Q}\right)^{2}\right)\right)^{1 / 2}$

where $f_{i}$ is the ith feature or index for case $\mathrm{C}$ or $\mathrm{Q}$, respectively, norm is a normalization factor, and $W_{i}$ is a weight attached to the ith feature. In some implementation of Formula (1), all fealures use the same weights. This will allow redundant, irrelevant, and other imperfect features to skew the similarity computation. Hence, many variants have been proposed to assign higher weights to more relevant features in a case base. After the similarity to the input query is computed for each case, a set of highly relevant candidate cases in a case base are scored. Cases with high similarity scores are presented to the user.

\subsection{HTN Guided Task Prioritization}

How does the agent select a task or group of tasks to perform next? This question can be answered with the help of the task network and the result of hypothesis ranking.

A task network provides a precedence constraint on the tasks. Horizontally in a task network, some tasks must be executed before others, while other tasks can be executed in parallel. Vertically in a task network, every task has a parent (except the root task), so there is a task and subtask relation. This relation allows the non-primitive tasks to be expanded, exposing the sub-tasks in a top-down manner.

In selecting a lask, there are several heuristics one can follow:

- a task should be selected only when its predecessors are satisfied;

- if there are several tasks in the same hierarchy that can be executed, a priority ordering can be imposed on the tasks such that a top priority task is able to affect the ranking of the case more than other tasks. 
- if a task is non-primitive, there may be several ways to achieve it according to a number of task reduction schemata. Thus there is a process of selecting an appropriate information source to answer a query, based on knowledge on the availability of the information source, the quality the potential information and reliability of the source, and the cost and duration of performing such as task.

\section{An Example}

Consider a scenario where a pilot flying a small airplane goes missing. A report comes in from the designation airport where the pilot was supposed to land indicating that the pilot has still not landed 2 hours after his expected arrival time.

Our situation assessment agent starts by accumulating information which will serve as a basis for hypotheses formation. The agent, based on the reported information about an overdue aircraft, will find all standard information such as time of day when the report was received, the weather report and so on. This information together with the knowledge that the plane is overdue is used to perform the hypothesis retrieval task from the case base.

Next, suppose that two hypotheses are retrieved, as shown in the figure 7 . These hypotheses are " $\mathrm{H}_{1}$ : plane crashed somewhere with serious darnage" and " $\mathrm{H}_{2}$ : plane landed somewhere to refuel." Both are possible hypotheses at this moment so they both have to be evaluated.

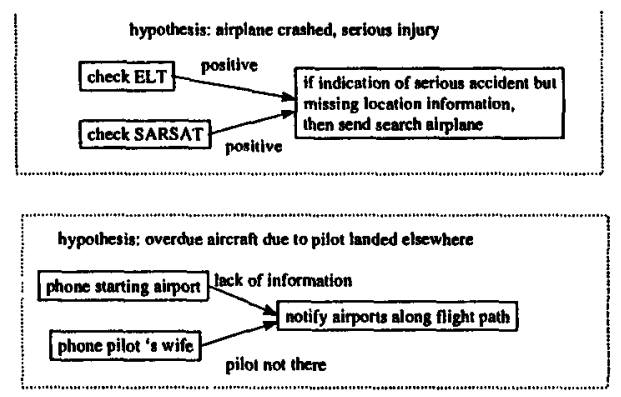

Figure 7: Two information seeking HTN's

The situation assessment (SA) is guided by the HTN's that are attached to the two hypotheses to be evaluated. According to the suggestions given by these task networks, there are four sub-tasks that can be immediately executed: check ELT signals, check SARSAT satellite reports, check airports along the flight path of the missing planc, and contact the pilot's wife for additional information. Of these four sub-tasks, the "check ELT" and "check SARSAT" sub-tasks can be carried out automatically by the agent, using monitoring agents attached to the ELT signal and SARSAT report databases. Suppose that at this point, both reports come back negative. The agent, based on similarity based ranking of the hypotheses objects, will decrease the likelihood of the planecrashed hypothesis.

The RCC controller, based on the information accumulated so far by the SA agent may proceed to phone the airport along the plane's flight path to get more information. Now, suppose that in the thick of things, the controller had forgotten to phone the pilot's wife. The agent would have sensed this not-yet performed task, and printed a friendly message to remind the controller to do that.

Suppose now that when talking to the pilot's wife, she reports that the pilot had called home. It now turns out that the hypothesis " $\mathrm{H}_{2}$ : plane landed somewhere to refuel" is a correct one in that the pilot did indeed decide to land in an intermediate airport in order to refuel, and while refueling, phoned home to tell his wife about the change of plan. The case is now closed with a report.

\section{Conclusions}

In this paper, we have presented an overall architecture for designing an intelligent agent for assisting RCC controllers in assessing situations related to an air incident. This system draws on several key technologies, including hierarchical task network planning, similarity based retrieval in case based reasoning and intelligent agents. The model relies on agents to monitor the actions of the human controller and the various information sources. At appropriate moments, the agent prompts the user for important steps that the human controller might have overlooked. 


\section{Acknowledgement}

The authors would like to thank the support from Canadian National Science and Engineering Research Council and Department of National Defense, DREV for support for the research.

\section{References}

[Cottam et al] Knowledge Level Planning in the Search and Rescue Domain, Hugh Cottam, Nigel Shadbolt, John Kingston, Howard Beck and Austin Tate. In Bramer, M.A., Nealon, R. (eds.) Research and Development in Expert Sysiems XII: Proceedings of BCS SGES Expert Systems'95, pages 309-326. SGES Publications. 1995.

[Cottam and Shadbolt] Knowledge Acquisition for Search and Rescue Hugh Cottam and Nigel Shadbolt

[Knoblock and Ambite, 1996] Agents for Information Gathering. Craig Knoblock and Jose-Luis Ambite. In Soltware Agents. Edited by Jeffrey M. Bradshaw. Pages 347-374.

[Lamontagne L., Benhamou P., Barat M. (1995)], COMODD - Melhods and Tools for decision making, Procecdings of the 1995 Workshop on Decentralized Intelligent Multi-agent Systems (DIMAS95), Cracovia, Poland.

[Leake 1996] Case Based Reasoning; Experiences, Lessions and Future Directions. Edited by David B. Leake. 1996 AAAI Press.

[NSM95] National SAR Manual Canada Dec 1995

[Perini and Ricci, 1996] An Interactive Planning Architecture: the Forest Fire Fighting Case. Anna Perini and Francesco Ricci. In New Directions in AI Planning. Edited by M Ghallab and A. Milani. 1996. Pages 273-283

[Tate 1977] Generating Project Networks. Proceedings of the IJCAI 1977. Pages 888-893. Morgan Kauffinan Publishers, 1977.

[Wilkins 1988] Praclical Planning: Extending the Classical Al Planning Paradigm. Morgan Kaur(man, 1988.

[Widom and Ceri 1996] Active Database Systems: triggers and rules for advanced database processing. Edited by Jennifer Widom and Stefano Ceri. Morgan Kaulfman Publishers. 1996

[Yang 1997| Intclligent Planning .-. A Decomposition and Abstraction based Approach. Qiang Yang. Springer Verlag. 1997 